\title{
VACUUM CONTROL SYSTEM FOR THE LOS ALAMOS LOW ENERGY DEMONSTRATION ACCELERATOR PROTON INJECTOR *
}

\author{
Leo R. Dalesio, Debora M. Kerstiens, LANL, Los Alamos, N.M., Mitchell C. Richards \#, WSRC, \\ Aiken, S.C., Gilbert A. Salazar, Joseph D. Sherman, David S. Warren, Thomas Zaugg, LANL, Los \\ Alamos, N.M.
}

\section{Abstract}

A vacuum control system has been developed for use on the Los Alamos National Laboratory continuous wave (cw) Low Energy Demonstration Accelerator (LEDA) Proton Injector. This paper summarizes hardware and software implementation to satisfy the following design criteria: (1) performs as a standalone system - no direct access to CPU/memory components by computer control hardware during normal operations, (2) has local (chassis) and remote control (EPICS [1] \& LabVIEW [2]) capabilities, (3) is electrically isolated and filtered from electrical transients created from injector high voltage spark-downs to ground, and (4) incorporates fast protect and fail safe components.

\section{GENERAL OVERVIEW}

The Los Alamos Low Energy Demonstration Accelerator (LEDA) project comprises a 75-keV proton injector, 6.7$\mathrm{MeV}$ cw RFQ, a high-energy beam transport system, and a high power beam stop [3]. The injector vacuum chamber, pump, and gauge system is composed of three diagnostic boxes (DB), each supplied with a turbo pump (TP), scroll pump (SCP), turbo pump gate valve (TGV), two vacuum ion gauges (VGI), and three vacuum convectron gauges (VGC). There are also two beam line gate valves (BGV), BGV1 located between DB2 \& DB3 and BGV2 located between DB3 and the radio frequency quadrupole (RFQ). Figure 1 is a drawing of the LEDA proton injector.

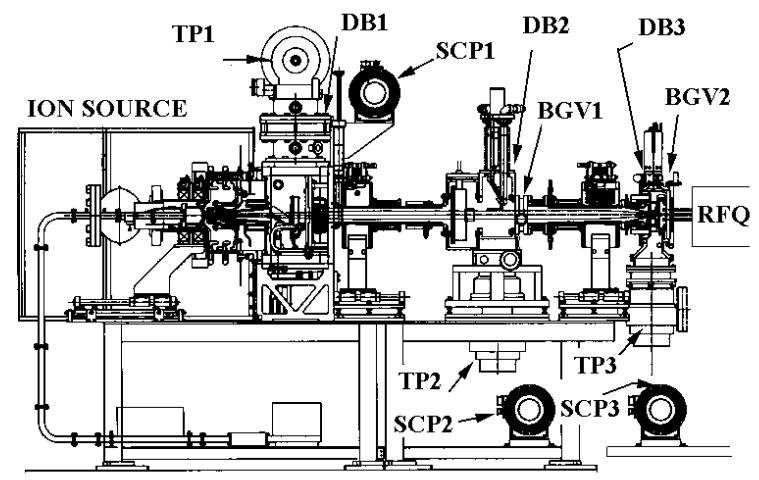

Figure 1. LEDA proton injector

\footnotetext{
* Work supported by the US Department of Energy

\# Email: mitch.richards@srs.gov
}

Figure 2 is a typical hardware configuration for the diagnostic boxes.

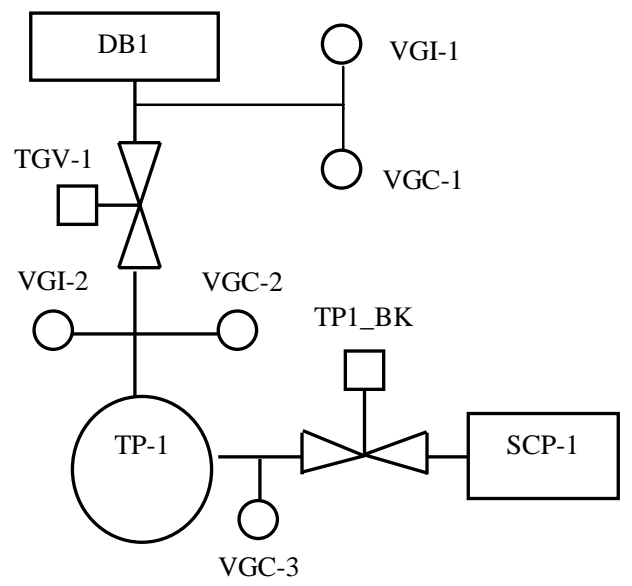

Figure 2. Typical vacuum hardware configuration for the diagnostic boxes.

The control system consists of one control chassis for each diagnostic box (e.g., Vacuum Pumping System \#1 \{VPS1\} for DB1, etc.), six ion gauge controllers, three turbo pump controllers, three motor starters, three current sensing relays, two programmable logic controllers (PLCs), electrical noise filters, and associated cable/wiring. A more detailed description of the system is discussed in the sections below.

\section{STANDALONE SYSTEM DESIGN}

The LEDA vacuum control system operates as a standalone system. That is, all hardware components are local to the injector, hardwired together, network isolated, and fully functional - contains all interlocks and requires no inputs from remote computer control equipment during normal operations.

Figure 3 shows a block diagram of the system devices. There are two Modicon [4] Compact PLCs involved (i.e., the Injector PLC and the EPICS PLC). The EPICS PLC is for remote operation and will be discussed in the next section. 


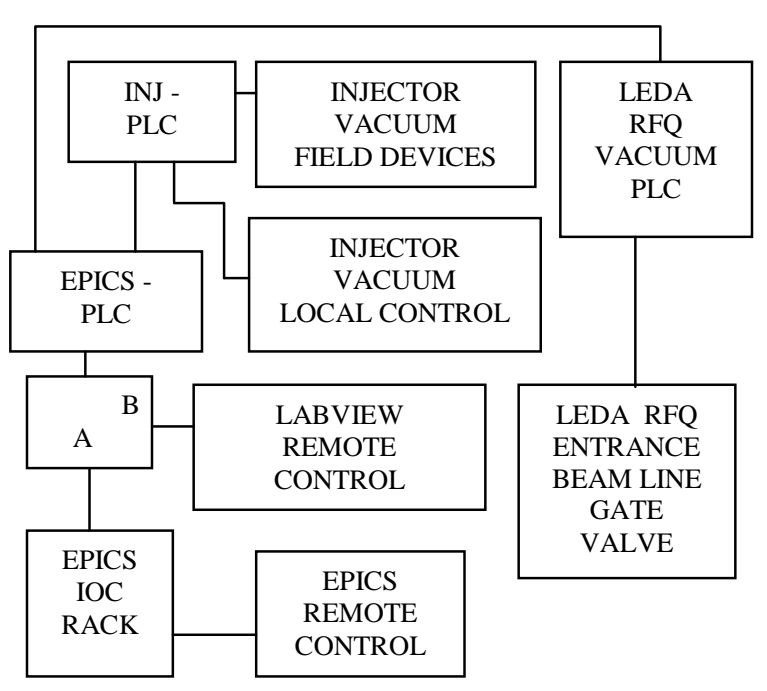

Figure 3. Block diagram of the LEDA Injector Vacuum Control System

The Injector PLC, however, processes all inputs and controls all outputs. There is no direct access to the PLC's CPU/memory components during normal operations. Thus, all signals are propagated through the associated input/output modules. A custom ladder logic program, containing all equipment interlocks, resides and runs (continuous loop) in the volatile memory of the CPU module. Of course, the program is lost during a power shutdown. There is, however, an Electrically Erasable Programmable Read Only (EEPROM) backup memory card installed in the CPU/memory module. Consequently, the ladder logic program is loaded and started shortly after power is resumed.

Control inputs are processed as requests. For example, if an operator desires to open TGV1 and presses the appropriate button on VPS1, the Injector PLC checks to see if all interlocks associated with TGV1 are enabled before opening the valve. Understandably, the PLC automatically shuts the valve, if during operations an associated interlock is disabled.

\section{LOCAL \& REMOTE CONTROL}

Local control is achieved by using three operator interface chassises. Figure 4 shows the front panel of Vacuum Pumping System \#2 (VPS2). Each chassis utilizes momentary push button switches and LED indicators. For example, if an operator wants to turn on turbo pump \#2, he/she would press the button residing just under the TP2 indicator. If all interlocks are enabled, turbo pump \#2 would turn on, verified by the illumination of the associated LED indicator. In short, the Vacuum PLCruns, as mentioned in section 2, a ladder logic program containing all the interlocks. Thus, the PLC makes the decision on whether or not to carry out an operator's request to actuate a device. The interlock chain will be further discussed in section 5 .

\section{VACUUM PUMPING SYSTEM \#2}

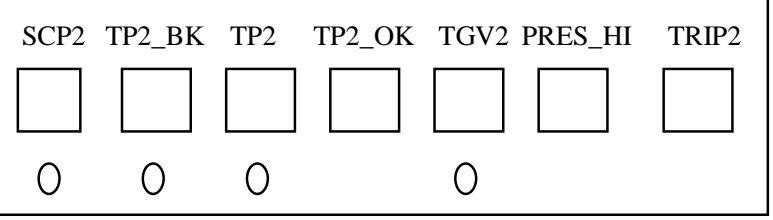

Figure 4. Front panel of VPS2

Remote control is achieved via the second Modicon PLC (i.e., EPICS PLC). It is called the EPICS PLC because it receives the remote operator requests via the EPICS network or a LabVIEW graphical interface. Basically, EPICS controls the LEDA injector vacuum control system by utilizing software screens, custom drivers, Input Output Controllers (IOCs), and two IOC modules. Those modules are a single board computer and a General Purpose Interface Bus (GPIB) card. The LabVIEW interface utilizes a personal computer, BusVIEW [5] (a Modbus [4] driver), serial port, GPIB board, and fiber optic cable \& communication modules.

Associated with EPICS remote control, a single board computer is loaded with a custom driver. That driver writes and reads memory locations (coils) residing in the form of a ladder logic program loaded in the EPICS PLC $\mathrm{CPU} /$ memory module. The coils are associated with input and output modules. When EPICS writes a binary 1, for example, into the PLC memory location represented by an output coil, the associated output module signal changes to the "on" state. That output signal (from the EPICS PLC) is wired to an input module of the Injector PLC. Thus, the Injector PLC interprets the incoming signal as a request to actuate the associated field device.

An EPICS read command works in the opposite direction. The Injector PLC outputs a signal representative of the state of a field device. For example, if beam line gate valve \#1 is open, the corresponding output signal is a binary 1 . That binary one is wired to an input module associated with the EPICS PLC. The ladder logic program running in the EPICS PLC has software contacts representative of input module signals. That is, if input signal one is in the "on" state, the associated software contact is closed. That software contact is in series with a coil (memory location) that changes to the "on" state when the inline software contact is closed. Thus, the EPICS network reads the state of that coil to determine the status of the corresponding field device. 


\section{ELECTRICAL ISOLATION AND FILTERING}

The LEDA injector vacuum control system is exposed to electrical transients created from injector high voltage spark-downs to ground [6]. Electronic equipment is known to fail from these short but damaging occurrences. To eliminate the negative effect of these electrical transients, multiple power supplies, relay isolation, and passive filtering are used. Figure 5 shows the typical electrical isolation and filter configuration for TGV1.

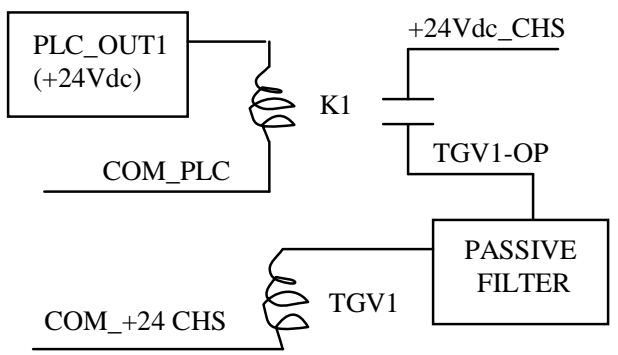

Figure 5. Electrical isolation and filter

Within each vacuum pumping system chassis, a power supply is mounted. The $+24 \mathrm{Vdc} \_\mathrm{CHS}$ is used to actuate vacuum pumps and valves, as well as read valve limit switches, corresponding to it's associated diagnostic box. That is, the power supply in Vacuum Pumping System \#1 controls and reads the field devices associated with DB1.

Mechanical relays are also used in each vacuum pumping system. They isolate the Injector PLC inputs and outputs (powered from the PLC supply) from the chassis supply driving the field devices. Consequently, the PLC supply voltage is better protected (since not distributed to the injector table) from the electrical transients produced from high voltage spark-downs.

Passive filters are used to protect equipment, as well. There are two stages. The first stage is a RC network. The second stage is a SEMIFILT [7] SP-H series distributed constant type noise filter, model SP333H.

\section{FAST PROTECT AND FAIL SAFE COMPONENTS}

Fast protect and fail safe operation are achieved by microwave control, monitoring hardware, and protective ladder logic.

The microwave power system provides energy to the plasma chamber to stimulate proton beam production. Not withstanding, if a beam line gate valve closes while the beam is on, damage is likely to occur. To protect the valves, a fast protect system is used. The fast protect components consist of a magnetron pulser chassis, signal interface module, and valve control interface box. Basically, the valve control interface box monitors the status of the valve's out limit switch. When the valve is open, the out limit switch is closed - allowing the passage of a voltage to the signal interface module. The signal interface module receives the valve open signal and sends a microwave enable signal to the magnetron pulser. The magnetron pulser controls the power to the microwave producing magnetron tube. Thus, the valve is protected, since microwave power to the plasma chamber is extinguished within a few microseconds after a valve closure is initiated.

Fail safe operation is accomplished by system monitoring hardware and protective ladder logic. Since the most vulnerable parts (as well as very costly) are the pumps (i.e., turbo \& scroll), monitoring hardware is installed. This hardware consists of flow meters, rotation relays, current-sensing relays, vacuum controllers, and overload devices. Interlocks from each of the devices are read by the Injector PLC and incorporated in the ladder logic program.

When a vacuum fault occurs, the turbo pumps are shut off and isolated - turbo gate and backing valves close. The scroll pumps (used for backing the turbos) continue to run, however, unless a current overload condition occurs. In either case, power is removed from the pumps, requiring fault correction and operator intervention before restart.

\section{CONCLUSION}

The LEDA Injector vacuum control system is robust. It operates as a standalone system, provides local \& remote control, is electrically isolated \& filtered from high voltage spark-downs, and incorporates fast protect \& fail safe components. In fact, the system has proven to be very reliable, with nearly a year of operation without failure.

\section{REFERENCES}

[1] Leo R. Dalesio, et. al., Nuclear Instruments \& Methods in Physics Research A 352 (1994), 179-184.

[2] LabVIEW®, trademark of National Instruments Corporation.

[3] J. David Schneider, these conference proceedings.

[4] Modicon ${ }^{\circledR}$, Modbus $®$, trademarks of AEG Schneider Automation, Inc.

[5] BusVIEW@, trademark of Soogatech Corporation.

[6] Michael E. Thuot, these conference proceedings.

[7] SEMIFILT®, trademark of SMI Electronic Devices America, Inc. 